\title{
Comercio inmigrante y economías étnicas: síntesis y críticas de los debates vigentes
}

\author{
Alejandro Garcés H.
}

Universidad Autónoma de Madrid, Madrid, España. Email: ajgarces@gmail.com

Resumen: El siguiente artículo presenta y discute los principales enfoques teóricos que han sido usados para comprender los nuevos negocios creados por inmigrantes en el marco de su inserción en las sociedades de destino, conformando lo que en la literatura especializada se ha dado en llamar "economía étnica”. A partir de la articulación de recursos de clase y étnicos traídos desde origen o consolidados en destino, y del peso que tiene el marco social, económico e institucional sobre la formación de estos nuevos emprendimientos, se revisan las principales dimensiones del fenómeno y los acentos de los enfoques que han predominado en su explicación.

Palabras clave: Economía étnica, negocio étnico, comercio inmigrante, migración internacional.

\section{Immigrant entrepreneurship and ethnic economies: summaries and reviews of current debates}

\begin{abstract}
This paper presents and discusses the main theoretical approaches that have been used to comprehend the new entrepreneurship created by immigrants in the process of insertion in host societies. These theoretical approaches are part of what has been named by the specialized literature as the "ethnic economy" theory. The paper will articulate the class and ethnic resources brought by the immigrants from the home society which are, in many cases, re-created in the host societies. It will also analyze the importance of the social, economic and institutional elements in the process of formation of these new entrepreneurship, reviewing the most important dimensions of the phenomena, and the approaches that have predominantly been used to explain it.
\end{abstract}

Key words: Ethnic economy, Ethnic entrepreneurship, Immigrants stores, International migration.

\section{Comércio de imigrantes e economias étnicas: resumo e críticas dos debates atuais}

Resumo: Este trabalho apresenta e discute as principais abordagens teóricas que têm sido usados para compreender as novas empresas criadas por imigrantes, como parte da sua integração nas sociedades de acolhimento, formando o que na literatura tem sido chamado de “economia étnica”. A partir da articulação de recursos de classe e étnicas trazidos de casa ou consolidadas no destino, eo peso que possui o marco social, econômico e institucional sobre a formação desses novos 
empreendimentos, revisamos as principais dimensões do fenômeno e os acentos das abordagens que têm dominado a explicação.

Palavras-chave: economia étnica, negócios étnicos, o comércio, a imigração, a migração internacional.

\section{Introducción}

El presente artículo aporta una revisión de las principales perspectivas y enfoques teóricos respecto a la formación y consolidación de los negocios étnicos o las economías étnicas en el marco de los emprendimientos comerciales desarrollados por poblaciones inmigrantes. Revisaremos como los principales autores del campo han caracterizado dichas economías en relación a los lugares en que ellas se verifican, a la manera en que se articulan con unos específicos proyectos migratorios y a la forma en que se determinan algunos elementos claves de la organización del proceso económico de los nuevos negocios. Nuestro objetivo aquí es resaltar cómo estas diferentes líneas teóricas articulan el incremento o reducción del flujo migratorio a una determinada localidad, unos proyectos migrantes de emprendimiento económico y la formación de unos espacios urbanos claves en la reproducción de la experiencia migrante en la ciudad.

A continuación revisaremos las principales perspectivas acerca de la noción de economía étnica, los diversos elementos que estos paradigmas ponen en juego, y a partir de ello la forma en que éstas nociones pueden adquirir cuando se las enfrenta a casos particulares. Como se verá, resulta fundamental aquí el encuadre entre los proyectos migratorios de los comerciantes étnicos (en cuanto a sus capitales sociales y educativos en los lugares de origen, sus trayectorias laborales previas al emprendimiento comercial que ahora les distingue, las motivaciones del emprendimiento que desarrollan), y las distintas estrategias y recursos que ponen en juego para la gestión de sus negocios.

En términos generales las teorías acerca de la noción de "economía étnica” o de "negocios étnicos", así mismo como la de "enclave étnico", tienen su origen o asiento empírico en los fenómenos migratorios que tienen lugar en los países desarrollados como sociedades de destino de los flujos de población. Trabajos pioneros como los desarrollados acerca del enclave cubano en Miami (Wilson \& Portes, 1980:51; Wilson \& Martin, 1982; Portes \& Bach, 1985; Portes \& Jensen, 1989) han marcado y son sintomáticos de la producción científica que con posterioridad, aunque con algunas diferencias que ya señalaremos, se ha venido desarrollando en Norteamérica y Europa. 


\section{Perspectivas críticas sobre la economía étnica y el comercio inmigrante}

La noción de economía étnica remite a la existencia de una actividad económica en la que los propietarios del negocio pertenecen a un específico grupo o colectivo étnico -o una minoría cultural o nacional señalarán otros autores (Portes \& Jensen, 1989:53)-, y que se dirige a una clientela preferentemente compuesta por miembros del mismo grupo de referencia. En este sentido, lo que a primera vista señala la idea de economía étnica es la configuración de un proceso económico que se cierra sobre sí mismo, un proceso en que tanto capital, trabajo y mercancías son puestos en circulación por parte de unos empresarios de origen extranjero, y que apuntan a proveer de un conjunto de bienes y servicios para un grupo étnico o migrante.

Así, podría entenderse que la economía étnica es un tipo de economía que se distingue por la fijación de una identidad cultural de quienes son titulares de estos negocios y su potencial clientela donde, como señalan algunos autores, los empresarios son aquellas personas dueñas de unos medios de producción que ponen en juego para extraer de ellos beneficio económico o un valor de mercado por los bienes y servicios que ofrecen. A partir de allí, la economía étnica o el empresariado étnico incorpora cualquier tipo de actividad económica que se encuentra fuera de la economía asalariada general (Beltrán, Oso et al., 2007:23).

En este sentido, la idea de la búsqueda de un beneficio económico podría hacer plausible aplicar esta definición a cualquier tipo de empresariado o agente económico. Sin embargo, pueden en primera instancia señalarse al menos dos elementos que apuntan en la dirección de fijar la especificidad de este tipo de economía: por una parte, la pertenencia del empresario a un grupo étnico o migrante; y por otra parte, la dependencia o la utilización de un conjunto recursos específicos, los llamados recursos étnicos, para el desarrollo de su actividad económica, esto es, una especie de acervo cultural propio del grupo migrante -ya precisaremos que tipo de elementos compondrían este capital- que vendría a ser rentabilizado en el desarrollo de la actividad económica que se trate (Light, 1972; Portes \& Jensen, 1989; Rath \& Kloosterman, 2000; Parella, 2005).

Sin embargo, el cierre que inducen este tipo de definiciones configura tan sólo un tipo ideal de economía étnica, compuesto por la propiedad del negocio por parte del individuo migrante, por el carácter co-étnico de su clientela, por la necesaria utilización de los llamados recursos étnicos (o con independencia de los modos en que éstos son utilizados) en la instalación y gestión de los negocios, y en definitiva por una restrictiva circulación en torno a una determinada identidad o minoría cultural. Todos estos elementos encorsetan o constriñen la definición de economía étnica. Desde nuestro punto de vista, este tipo de rigideces inhibe la posibilidad de dar cuenta de la diversidad de formas que presenta lo comercial en espacios 
urbanos dinamizados por la presencia de los inmigrantes (Garcés, 2006, 2007, 2010), invisibilizando las diversas formas en que se organiza el trabajo, los modos en que se mercantiliza lo étnico o lo migrante (Werbner, 1999), la forma en que se definen las orientaciones comerciales o las clientelas, entre otros elementos. Si bien, esta noción de economía étnica ilumina una serie de procesos que tienen lugar en las comercios migrantes, es necesario apuntar que el concepto es poco eficiente para analizar la manera como estos comercios se encuadran en proyectos migratorios específicos, impactando la forma con que los migrantes dialogan con lo local-nacional en su inserción urbana, y cómo, a través de esta nueva economía, múltiples agentes trabajan o reproducen lo "étnico" o lo "nacional” de los propios migrantes.

En la perspectiva de relativizar la definición de economía étnica, puede sernos útil al menos en primera instancia la distinción elaborada por Light y Gold entre una economía de propiedad étnica y otra étnicamente controlada. La primera constaría de aquellos emprendimientos en que el inmigrante cuenta con la propiedad del negocio en cuestión, mientras que en el segundo tipo la propiedad no es imprescindible mientras los empleados extranjeros, co-étnicos o inmigrantes en general, mantengan un poder de mercado (importante y duradero dirán los autores) sobre los lugares de trabajo, ya sea por su cantidad, concentración y/o organización. El ejemplo que aquí dan los autores sería el de un gremio, en la medida de que éstos influirían en las políticas de contratación y salarios (Light \& Gold, 2000:23; Light, 2007:41-42). Esta distinción aporta una importante herramienta analítica en la medida en que relativiza el tema de la propiedad de los negocios, permitiendo ampliar la perspectiva en al menos dos sentidos.

Por una parte, posibilita una lectura de la economía étnica como un espacio del que participan otros actores más allá de quienes son titulares de los negocios. Nos referimos tanto a los familiares que aportan su fuerza de trabajo en empresas familiares, como a aquellos que se desempeñan como trabajadores asalariados. La importancia de esto radica en que podemos concebir los espacios comerciales como campos de relación del colectivo migrante, como lugares de acceso a recursos, como escenarios de una cierta movilidad social, más allá de comprenderla únicamente como una estrategia individual de emprendimiento del titular del negocio. En esta perspectiva se destaca las definiciones de economía étnica de Bonacich \& Modell (1980:45) y Light \& Gold (2000:9), quienes precisamente hacen hincapié en la diversidad de actores que participan del proceso económico en cuestión, afirmando que una economía étnica estaría compuesta por auto-empleados de cualquier grupo étnico o inmigrante, sus empleadores, sus empleados co-étnicos, y sus trabajadores familiares no remunerados.

Por otra parte, la distinción nos permite explorar otras "formas de ser” de la economía étnica, discutiendo por ende su principal descriptor, el carácter étnico. En este sentido, podemos explicar, por ejemplo, la emergencia de comercios cuyos titulares son pequeños empresarios nativos den- 
tro de espacios urbanos masivamente ocupados por comercios migrantes, o donde la residencia migrante se concentra ¿Puede ser esto concebido como parte de una economía étnica? En principio no, dado que la propiedad del negocio no es étnica sino autóctona, y que tampoco es co-étnico a los propietarios de los establecimientos el colectivo de personas que trabaja en dichos espacios, o su clientela. Sin embargo, se trata de unos espacios laborales ocupados privilegiadamente por migrantes, su clientela es fundamentalmente migrante, $y$, elemento no menor, su emplazamiento en el territorio urbano los hace formar parte sustancial de unos espacios que podríamos entender "etnificados", espacios donde se recrean ámbitos de sociabilidad e identidad propios del colectivo migrante (Torres Pérez, 2008:391) ${ }^{1}$. En este sentido, si distinguimos entre el enclave étnico como la expresión espacial de la concentración de población inmigrante, y la economía étnica como el proceso económico que le es interno, ¿podríamos decir que estamos ante un enclave étnico sin economía étnica? Pues bien, por lo pronto diremos que es posible identificar formas diversas de participar de las dinámicas de los espacios urbanos en que los negocios migrantes normalmente se concentran, que ponen en cuestión la pertenencia étnica o migrante como condición sin equa non de su caracterización. Con todo, no queremos con esto dar la idea de que la presencia de pequeños empresarios autóctonos en estos espacios sea algo predominante, sino más bien, subrayar que su permanencia implica una sustancial complejización de las dinámicas internas en los enclaves migrantes.

Otro elemento importante para ampliar la perspectiva respecto de la noción de economía étnica dice relación con la formalidad de los emprendimientos comerciales que pueden observarse. Tomando este elemento en consideración, algunos han distinguido una economía étnica compuesta de al menos tres subsectores: formal, informal e ilegal (Tienda \& Raijman, 2000), al interior de los cuales puede presentarse tanto una economía de propiedad étnica como una étnicamente controlada. Ahora bien, ¿qué tipos de emprendimiento compondrían estos subsectores?

El subsector formal incluiría a las empresas que pagan impuestos y están registradas oficialmente. Se trata del sector más profusamente estudiado en economía, donde los propietarios y los empleados pertenecen ambos al mismo colectivo extranjero o inmigrante. El subsector informal constaría de empresas que, produciendo bienes y mercancías legales, lo hace sin pagar impuestos ni tener reconocimiento oficial (Bourgeois, 1995). El subsector ilegal, por su parte, lo conformarían empresas que manufacturan o distribuyen productos o mercancías prohibidas a clientes que las requieren, especialmente drogas, juego y prostitución, pero también documentos falsos de inmigración, pornografía, videos piratas (Light, 2007:43) y, crecientemente en las ciudades europeas, la falsificación de productos asociados a grandes marcas de ropas, calzados y accesorios.

De cualquier modo, lo que en teoría y comúnmente distinguiría a la economía étnica sería la dependencia en parte de un capital proporcionado por los recursos étnicos, que a su vez pueden agrupar elementos como: 
“...el valor de la lealtad y la confianza, los lazos de solidaridad y reciprocidad, el empleo de coétnicos, la ayuda familiar, las facilidades de préstamo de dinero por parte de familiares, amigos y vecinos, la socialización étnica y lingüística en determinados valores y actitudes, así como el peso que juegan las tradiciones y estrategias económicas étnicas (a menudo vinculadas a los lugares de origen)... Una característica bastante común a este tipo de negocios es la utilización de un tipo de empresa de corte familiar.” (Beltrán, Oso et al., 2007:27-28)

El tema de la utilización de los recursos étnicos, su rol o carácter determinante en la configuración de las economías étnicas nos lleva a la discusión entre las aproximaciones teóricas predominantes de este tipo de economías en contexto migratorio. Si bien el debate a este respecto es variado y en algunos aspectos hasta confuso, existe relativo consenso en distinguir entre perspectivas de orden culturalista, aproximaciones ecológicas, perspectivas estructuralistas o que hacen énfasis en el marco de oportunidades que ofrecen las sociedades de destino del flujo migratorio, y perspectivas interaccionistas o modelos más bien integradores de las otras aproximaciones. En términos generales, puede adelantarse que las diferencias entre las distintas perspectivas radican en el peso específico de que suele dotarse a los recursos étnicos.

\section{Las perspectivas culturalistas}

Los primeros énfasis de la investigación en economías étnicas estuvieron marcados por las aproximaciones que ponían acento en los factores étnico-culturales a la hora de explicar la formación de estos nuevos negocios étnicos o inmigrantes. El nudo central en esta perspectiva, esencialista en cierto modo, es que habría una afinidad opcional (Arjona, Checa et al., 2007:2) en algunos colectivos que tenderían hacia el autoempleo, ya sea por una tradición comercial o por cuestiones de índole religiosa, todos a su vez elementos que marcarían el éxito y fracaso de las empresas. En este sentido, se determinaría un patrón de incorporación a la sociedad de destino del flujo migratorio [piénsese en la teoría de los modos de incorporación (Portes \& Böröcz, 1998)], que operaría como una respuesta cultural a la hostilidad de un entorno caracterizado por las segmentación del mercado de trabajo. ${ }^{2}$ Este tipo de lógicas es la que sustenta la ya clásica teoría de las minorías intermediarias (Bonacich, 1973), que argumenta que los grupos establecen redes de solidaridad intragrupal para el sostenimiento de los negocios y una consecuente o supuesta auto explotación del entorno familiar, y en general de co-étnicos asalariados, como una forma de reducir los costos que conlleva la actividad o la precariedad y riesgos de la misma.

Siempre en el marco de las aproximaciones que intentan situar la relevancia de los factores étnico-culturales en el desarrollo de estas economías, resulta interesante la distinción de perspectivas que realizaban Portes y Jensen a finales de la década del 80, ya que allí era caracterizada como 
pesimista la pionera aproximación de Bonacich. La crítica de estos autores se basaba en la noción de que las empresas étnicas y las comunidades de emprendedores podrían ser comprendidas en términos positivos: como un no ortodoxo pero efectivo vehículo de movilidad para estas minorías (Portes \& Jensen, 1989:930). Las comunidades étnicas ofrecerían acceso a recursos (créditos, mercados y trabajo), que permite a las pequeñas empresas sobrevivir y prosperar (Wilson \& Martin, 1982; Nelson \& Tienda, 1985; Portes \& Bach, 1985).

Estos autores han profundizado esta crítica argumentando que este tipo de emprendimientos permiten adaptaciones funcionales a la ausencia de empleo regular, dado el contexto hostil en las sociedades receptoras (por ejemplo, la discriminación en el mercado de trabajo). Es legitimo afirmar que el énfasis de esta segunda escuela habría cambiado gradualmente desde la función de sobrevivencia de la temprana empresa inmigrante a una más positiva caracterización de tales iniciativas como medios de ascenso económico para la población inmigrante (Light, 1984; Waldinger, 1986). En este sentido, la adaptación funcional se fundaría en la idea de que el factor cultural suspendería o aislaría estas iniciativas económicas del entorno hostil en que se encuentran. Como señala Cebrián y Bodega en el marco de un estudio acerca del caso de los comercios inmigrantes en Madrid,

“...el factor cultural anula las condiciones materiales y propicia un comportamiento económico que se desvía de la norma: los atributos culturales de ciertos grupos minoritarios explican y determinan la tendencia a la inversión y a la creación de negocios propios. Las comunidades asiáticas son presentadas, a menudo, como ejemplos paradigmáticos.” (Cebrián de Miguel \& Bodega, 2002:564).

Por último, se ubicaría la perspectiva descrita como más pesimista de Bonacich, para quien este tipo de formas económicas serían un vehículo de explotación capitalista. Las grandes empresas capitalistas usarían emprendedores intermediarios para explotar la mano de obra inmigrante cautiva y reducir sus propios costos de producción. Las condiciones laborales son descritas en las empresas étnicas como similares a aquellas del más amplio mercado laboral secundario, ofreciendo trabajos precarios. Pueden encontrarse allí en una peor situación, con propietarios que invocan las cadenas étnicas y toman ventaja de la ignorancia de los migrantes en cuanto al idioma y a las leyes laborales para imponer duras condiciones de trabajo (Bonacich, 1987; Sanders \& Nee, 1987).

La crítica a este modelo explicativo que pone acento en los factores étnico-culturales se funda en rechazo a esta supuesta "burbuja” que supondrían las economías étnicas, entendidas desde los enfoques culturalistas como en una especie de suspensión de la actividad comercial respecto del contexto social y económico del que termina formando parte. En síntesis, la crítica apunta a la reducción del emprendimiento inmigrante a un fenómeno etno-cultural dentro de un vacío económico e institucional (Rath \& 
Kloosterman, 2000:666). Sin embargo, a partir de esta crítica se introduce una distinción bastante útil a los efectos de la descripción concreta del proceso que hay detrás de la noción de economía étnica. Se trata de la distinción entre recursos étnicos y recursos de clase, como factores que incidirían siempre en la formación de los nuevos emprendimientos pero de manera diferenciada.

Los recursos étnicos serían el conjunto elementos o factores socioculturales que estarían a la base del surgimiento y sostenimiento de los negocios étnicos. Se trataría del uso de las redes sociales étnicas para la transmisión rápida de información, orientación económica a los empresarios y a quienes pretenden llegar a serlo, así como el acceso rápido a mano de obra barata (Light \& Rosenstein, 1995). Podrían entonces distinguirse recursos étnicos de orden material (relativos a la formación), informativos (consejos), o basados en la experiencia. De este modo, los recursos étnicos incluirían las relaciones de amistad, los sistemas de matrimonio, las relaciones de confianza, el capital social, los factores culturales, la religión, la lengua, los valores y actitudes empresariales, las asociaciones de crédito rotativas, la solidaridad, las redes sociales y la ideología de solidaridad étnica ${ }^{3}$ (Solé \& Parella, 2005:53). Otros autores entenderán por recursos étnicos un conjunto de prácticas etno-culturales, ideologías étnicas, redes sociales e instituciones étnicas (Light \& Gold, 2000). Por su parte Rath y Kloosterman, en su revisión teórica para el caso holandés han observado que estas características han sido de manera predominante las tradiciones del grupo, las estructuras morales, patrones de comportamiento y las lealtades étnicas (Rath \& Kloosterman, 2000:666).

En paralelo a los recursos étnicos tendríamos los recursos de clase, entendidos como distintas formas de capital en posesión de un grupo, ya sea éste financiero, humano, cultural o social (Light \& Gold, 2000:83), o lo que en la perspectiva de Solé y Parella (2005) englobarían atributos culturales y materiales. Los primeros relacionarían con la cultura profesional compuesta por valores, actitudes, conocimientos, saberes y habilidades transmitidas en el transcurso de la socialización, mientras que los materiales darían cuenta de la propiedad de los medios de producción, el patrimonio, el capital humano, dinero para invertir, etc., elementos que se entenderán como propios de cualquier burguesía (Solé \& Parella, 2005:53).

A partir de allí, los estudios podrían inscribirse por sus acentos en una u otra perspectiva. A este respecto Arjona y Checa han señalado que los trabajos de Portes y Zhou por ejemplo recurren más a cuestiones que tendrían que ver con el capital social (en términos de redes y solidaridad intragrupal), más que a la tan citada tradición comercial o empresarial en origen. O también el caso de Fairlie y Meyer que ponen en duda la correlación entre tradición empresarial y autoempleo en destino, o lo que es lo mismo, no habría una afinidad inicial ni en la forma (autoempleo) ni en el sector de dedicación (Arjona, Checa et al., 2007:3). Por el contrario, como se ha visto en investigaciones acerca del caso del colectivo chino y senegalés en Cataluña, la incidencia de las tradiciones comerciales en origen resultan 
fundamentales para entender los patrones de inserción de estas poblaciones (Beltrán, 2000; Sow, 2004). Con todo, las investigaciones más recientes intentan compatibilizar ambas perspectivas, en la medida también de que la articulación entre recursos étnicos y recursos de clase puede ser útil a la hora de diferenciar los emprendimientos inmigrantes de los emprendimientos de comerciantes autóctonos en el mismo tipo de negocios.

En la perspectiva de postular un equilibrio entre ambos tipos de recursos se encuentra el trabajo de Light y Gold. Para ellos los recursos de clase promueven la expansión, la introducción en los mercados de masas, pero los recursos étnicos son esenciales para el comienzo del negocio y para la misma inmigración (Light, 2007:58). Esto se relaciona a su vez con los tipos de redes que pueden articularse al servicio de los nuevos emprendimientos. Por una parte, se distinguen unas redes de vínculos fuertes que incluyen a los amigos más cercanos y a familiares, y por otra, se observan redes de vínculos débiles que incluyen a amigos ocasionales o conocidos. Así para Light, los

“(...) vínculos fuertes y los comienzos dependen de los orígenes étnicos, pero el acceso a información y la expansión requieren contar con los recursos de clase. Estos temas sitúan a la investigación de la economía étnica en conexión con los procesos básicos por los cuales el capitalismo transforma las sociedades, incluyendo cómo, dónde, y por qué ocurre la asimilación de los inmigrantes... la economía étnica existe como una esfera separada de la actividad capitalista que requiere un análisis diferenciado. Lo que no está claro es hasta qué punto son diferentes las normas que gobiernan el funcionamiento interno de la economía étnica de las que gobiernan las de la sociedad general.” (Light, 2007:58-59).

\section{La perspectiva ecológica}

Esta línea de investigación acerca de las economías étnicas -y en algunos casos sobre las denominadas "economías de enclave" - ha puesto su acento en los procesos de sucesión y/o sustitución de población residente y de negocios en el espacio urbano, a partir de la actualización de un determinado flujo migratorio. En términos generales, lo que se constata es el abandono progresivo de la población autóctona de unos nichos de negocio para incorporarse a mercados más globales y rentables. Este proceso, a su vez, da lugar a una recomposición étnica de los barrios en que la migración tiene lugar, y terminan produciendo una clara modificación del paisaje urbano. Se entiende que resultan aquí fundamentales las necesidades de provisión de bienes y servicios específicos por parte de la población migrante que se instala.

Dentro de este enfoque es interesante el movimiento que va desde los análisis que se centran en la sucesión en el área residencial hacia la sucesión en la propiedad de los negocios (Aldrich, 1975; Aldrich \& Reiss, 1976). Como señalan Arjona y otros, uno de los trabajos más significativos 
acerca de estos procesos es el que desarrollaron Aldrich y Reiss, quienes estudiaron el paso del control de las empresas de la economía local desde los empresarios autóctonos hacia los nuevos empresarios inmigrantes puertorriqueños en tres ciudades norteamericanas. Así, constataron el proceso de sustitución dando cuenta que de que la proporción de comerciantes blancos se reducía mientras simultáneamente aumentaba el porcentaje de empresarios negros puertorriqueños. Por otro lado, se constataba un desplazamiento de los empresarios blancos a zonas donde la presencia inmigrante era menor y los beneficios económicos de sus emprendimientos eran mayores, mientras los inmigrantes imposibilitados de acceder a lugares con mayor valor social y económico, se ubicaban en los lugares abandonados por los autóctonos, de donde obtienen una rentabilidad que ya no existía para los primeros (Arjona, Checa et al., 2007:3-4).

En el caso español el estudio de estos procesos de sustitución aparece como un elemento emergente a propósito de investigaciones más generales sobre la formación de economías étnicas, de procesos de generación de barrios inmigrantes, de procesos de inserción urbana de población inmigrada, y de construcción de espacio público. Destacan en este sentido, los trabajos acerca del caso barcelonés (Moreras, 1999; Aramburu, 2002; Monnet, 2002; Solé \& Parella, 2005; Serra, 2006), del caso almeriense (Arjona, 2006; Arjona \& Checa, 2007), o del caso madrileño (Buckley, 1998; Cebrián de Miguel \& Bodega, 2002; Garcés, 2005, 2006; García Ballesteros, García et al., 2006)

En el caso chileno, nuestra investigación sobre la migración peruana en la capital Santiago, observa cómo los espacios que concentraban los comercios migrantes resultaban de un proceso de sustitución comercial, sobretodo en calles como Catedral (en la comuna de Santiago Centro), donde la desaparición del comercio local o nativo, básicamente empresas del ámbito textil, ocurrió paralelamente a la emergencia comercial inmigrante en la zona (Garcés, 2007:8). Un contraste interesante planteaba a su vez el que no fuera posible certificar el mismo fenómeno en otros espacios ocupados por comerciantes peruanos, como en los alrededores de calle Rivera en la comuna de Independencia, donde no existía previamente ese tejido comercial local o nativo a suceder o sustituir, sino que es la propia fuerza de la migración, con sus necesidades de acceso a bienes y servicios específicos, la que genera las condiciones para la emergencia del comercio inmigrante (Garcés, 2010). También es reseñable aquí, si bien no aborda directamente las dimensiones espaciales del fenómeno, los trabajos de Stefoni acerca de la del surgimiento de negocios inmigrantes organizados en torno a la gastronomía peruana y su consolidada aceptación entre la población nativa (Stefoni, 2005, 2008).

\section{El énfasis en la estructura de oportunidades}

Desde esta perspectiva se entiende que el uso de los recursos étnicos y los recursos de clase, núcleo de las economías étnicas, están determina- 
dos por la estructura de oportunidades que ofrecen las sociedades en que se insertan los colectivos de emprendedores inmigrantes. En este sentido, el enfoque se instala sobre la crítica a las aproximaciones culturalistas, enfatizando que éstas no prestarían suficiente atención a las condicionantes económicas y situacionales en que tienen lugar los nuevos negocios. Para Solé y Parella,

“(...) la creación de estos negocios étnicos es entendida, desde estos planteamientos, como una reacción al bloqueo de oportunidades en el mercado de trabajo; de manera que la autoocupación se erige como una estrategia de supervivencia vinculada a los lazos de solidaridad existentes dentro de la comunidad.” (Solé \& Parella, 2005:55).

Como se ve, no es que desde esta perspectiva no se considere la actuación de los recursos internos propios al colectivo. Lo que se subraya más bien, es su subordinación al contexto social, económico y cultural en destino. En esta línea puede comprenderse también la noción de etnicidad reactiva, frente a la situación desventajosa de entrada al mercado laboral, o en términos generales de incorporarse a la economía principal. Sin embargo, esto puede llevarnos de manera demasiado rápida a pensar las economías étnicas como estrategias de refugio, como única salida, o como salida involuntaria frente a los obstáculos señalados, lo que no se produce necesariamente así, al tiempo que inhabilita pensar estos espacios como motores de una cierta movilidad social.

Los trabajos de Min acerca de la empresarialidad coreana en Estados Unidos en los años 70, (Min, 1984, 1996), son usados por Solé y Parella para dar cuenta del carácter fundamental de estos factores situacionales en la producción de las economías étnicas. Básicamente en sus trabajos se constata el hecho de que la concentración de negocios étnicos se explica por las desventajas en el mercado de trabajo, sobre todo para quienes se encuentran en primera instancia con el obstáculo del desconocimiento de la lengua local. Luego, contra la asentada afirmación de que sería la tradición cultural confuciana y el acervo cultural protestante en el caso de estos migrantes lo que les llevaría al establecimientos de estos negocios -fruto a su vez de una particular ética del trabajo y del establecimiento de vínculos familiares-, para Min la explicación de la elevada tasa de auto-ocupación de este colectivo debe buscarse en los factores estructurales y situacionales, los que determinarán una actividad comercial caracterizada por unas largas jornadas laborales y por el trabajo no remunerado de la familia (Solé \& Parella, 2005:57).

Sin embargo, ¿qué otros elementos, además del conocimiento de la lengua y la discriminación en el acceso a empleo, componen una estructura de oportunidades que favorece la emergencia de las economías étnicas? La investigación de Gómez acerca del comercio inmigrante en la ciudad de Madrid, aporta algunas luces a este respecto. Intentando dar cuenta de las oportunidades y limitaciones que ofrece la ciudad en un contexto de cambios demográficos, socioeconómicos y culturales, la autora distingue pro- 
cesos que podrían conformar una estructura de oportunidades para las economías étnicas en la ciudad (Gómez, 2006:45-49, 2007:2-4):

- Proceso de envejecimiento de la población que acarrea impactos en lo comercial al producir una reducción considerable de las ventas, dado que la vejez supone en general menor poder adquisitivo y menor nivel de consumo. La vejez, al implicar una reducción de la movilidad, afectaría a los lugares en que se realizan las compras, tendiendo a realizarse éstas en supermercados y pequeñas tiendas de alimentación cercanas al hogar que comparten la característica de 'tener de todo', o también el servicio a domicilio.

- Desplazamiento de la población debido al acceso a la vivienda. Las familias jóvenes trasladan su residencia hacia otros barrios periféricos y ciudades en busca de mejores relaciones calidad-precio de la vivienda, lo que hace que envejezca la población de algunos barrios y por lo mismo baje el consumo. Además hay un relevo de población con el abandono por parte de autóctonos y la llegada de inmigrantes, produciendo a su vez un relevo de la clientela.

- Tendencia a la generalización del trabajo remunerado de las mujeres fuera del hogar. Esto provocaría un cambio en los ritmos comerciales, ya que las compras ya no se realizaría todos los días ni en los mismos horarios.

- Trasformación de los horarios laborales. Prolongación del tiempo y días dedicados al trabajo.

- Cambio en las expectativas laborales, acarreando un menor relevo del pequeño empresariado comercial local. En este sentido, los hijos no siguen el camino de los padres dada las dificultades de conciliar la actividad con la vida familiar y social.

- Surgimiento y consolidación de otras fórmulas comerciales. Expansión de supermercados, centros comerciales, y/o compra por Internet, franquicias, que consiguen mejores precios gracias a la estrategia del pago a largo plazo a los proveedores, horarios más amplios, innovaciones técnicas o complementarias (pago con tarjetas, servicio a domicilio), que han implicado una fuerte competencia para el comercio tradicional, y una oportunidad para el comercio inmigrante que ha venido sustituyéndolo.

- Tendencia a la normativización por parte de las administraciones públicas en cuestiones como higiene, seguridad y protección del consumidor. El tema más conocido y controvertido es el de los horarios comerciales.

Más allá de las especificidades que presenta el estudio de Gómez, lo que interesa resaltar es la construcción de un contexto para la emergencia 
del comercio inmigrante, un contexto que más allá de los recursos étnicos y de clase de los migrantes, determina el panorama del pequeño comercio en las sociedades de destino del flujo migratorio.

\section{Los modelos interaccionistas o integradores}

En términos generales podemos decir que las perspectivas interaccionistas intentar mediar o establecer una interconexión entre los recursos internos de las comunidades migrantes y la estructura local de oportunidades (Kloosterman, Van der Leun et al., 1999; Rath \& Kloosterman, 2000; Kloosterman, 2001). Se trataría de un enfoque más integrador en la medida en que el objeto de análisis no serán de manera aislada los informales modos de gestión de los recursos por parte de los nuevos comerciantes, ni tampoco las estructuras económicos sociales de la sociedad de destino.

Según Solé y Parella, en el marco de este enfoque podrían distinguirse tres grupos de elementos que debiéramos entender actuando de manera simultánea. En primer lugar tendríamos las características del grupo, compuesta de su capacidad para movilizar recursos informales, solidaridad familiar o comunal. En segundo lugar, la estructura de oportunidades, que englobaría tanto las condiciones de mercado (consumidores de productos étnicos, mercados abiertos no étnicos) como las condiciones de acceso a los negocios (vacantes, políticas gubernamentales, etc.). Por último, las estrategias étnicas darían cuenta del uso de los recursos en las estructuras de oportunidades.

"Las estrategias étnicas emergen de la capacidad de adaptación de los empresarios inmigrantes al contexto en que se insertan, mediante la movilización de los recursos disponibles. Los negocios étnicos son, pues, la articulación de estos tres conjuntos de variables: características del grupo, estructura de oportunidades y estrategias étnicas.” (Solé \& Parella, 2005:58).

Estas estrategias étnicas serán particularmente importantes no solo en la organización interna de los negocios, en la forma en que articulan familia, capital y trabajo, sino también en la forma en que se organiza su emplazamiento en la ciudad, en la eventual formación de barrios de migrantes, y en definitiva en una concreta apropiación del espacio urbano.

Pues bien, en el modelo de Waldinger, Aldrich y Ward (1990), que podrían considerarse representantes de esta perspectiva interaccionista, los negocios étnicos serían entonces consecuencia de una estrategia étnica determinada por las características del grupo y la estructura de oportunidad. En la caracterización del grupo étnico o migrante se parte nuevamente de la situación de desventaja en el mercado laboral que caracteriza a los colectivos migrantes, un mayor nivel de aceptación de un puesto de trabajo -dado el diferencial salarial con los países de origen, y de la supuesta menor aver- 
sión al riesgo por parte de unos inmigrantes más preocupados de la movilidad económica que del status social (Waldinger, Aldrich et al., 1990:33; Solé \& Parella, 2005)-. Sin embargo, habría que revisar el supuesto escaso status social que tendrían este tipo de emprendimientos comerciales, que quizás sea efectivo en el marco de la sociedad de destino, pero que al interior del colectivo puede ser sometido a otro tipo de valoraciones.

Dentro de las características del grupo étnico, los autores distinguirán entre los factores de predisposición y la movilización de recursos. Los factores de predisposición estarían compuestos por las aspiraciones de los migrantes, su reacción ante la situación de bloqueo a la movilidad social y económica en origen, su conocimiento del idioma, su formación empresarial (nótese aquí la referencia a los recursos de clase que señalamos más arriba, el carácter permanente temporal o permanente de la migración, etc.) (Waldinger, Aldrich et al., 1990:32-33). En el caso de la movilización de recursos, se refiere por una parte a la utilización de los lazos entre coétnicos para proveerse de mano de obra barata, hasta el punto en que los comercios mismos se trasforman en el germen de nuevos proyectos migratorios. En general, se entiende que estas redes remiten a los lazos más estrechos entre los migrantes, fundamentalmente de orden familiar, aunque sin embargo la movilización de recursos remite a las redes sociales más amplias donde es posible acceder por ejemplo a algunos sistemas de crédito, centrales a la hora de establecer los negocios. Por último, refiere también a los recursos que son posibles de utilizar a partir de las políticas públicas respecto de las minorías étnicas que se encuentren en vigor en las sociedades de destino, como ha sido posible observar en algunos momentos en Estados Unidos e Inglaterra fundamentalmente (Waldinger, Aldrich et al., 1990:34-40).

En el marco de las estructuras de oportunidad, se distinguirán al menos dos ámbitos: las condiciones del mercado y el acceso a la propiedad de los negocios. Las condiciones del mercado se encontrarán determinadas tanto por el potencial y efectivo consumo de productos étnicos, como por la existencia de mercados abiertos no étnicos. Este tipo de mercados correspondería a aquellos sectores económicos no atendidos o abandonados por los comerciantes nativos, o aquellos sectores en que la contraparte nativa también está presente, pero donde el comercio étnico provee otros servicios, como por ejemplo jornadas de atención más largas, disponibilidad de crédito, ofertas o venta en pequeñas cantidades (Waldinger, Aldrich et al., 1990:25-26).

En este marco de negocios dirigidos al conjunto de la población, Solé y Parella distinguen cuatro posibilidades:

- Mercados situados en nichos marginales. Remiten básicamente a los ámbitos de negocios desatendidos o rechazados por los comerciantes autóctonos, mercados con unas bajas economías de escalas y gran número de competidores, y donde los comerciantes inmigrantes por lo general basan su competitividad en la extensa duración de la jornada laboral. Ejem- 
plos en este sentido los encontramos en la industria del taxi en Nueva York o los hornos de pan en Ámsterdam (Waldinger, Aldrich et al., 1990).

- Negocios vinculados a la aparición de la sociedad posindustrial y su creciente demanda de servicios. Se trata de oportunidades de negocios a pequeña escala para los comerciantes inmigrantes, de baja tecnología, intensivos en fuerza de trabajo, pero a su vez de baja cualificación (Sassen, 1991). Pensemos por ejemplo en el caso de los centros de llamados telefónicos e Internet.

- Mercados de productos segmentados. Dada la inestabilidad e incertidumbre de la demanda (sectores menos competitivos de la producción), las empresas inmigrantes se orientan a productos que las grandes empresas no pueden ofrecer, principalmente en los sectores de la construcción y confección.

- Mercados de productos exóticos. Éste último tipo de mercado resulta de singular importancia en el caso de la economía étnica peruana que estudiamos en Santiago de Chile (Garcés, 2007), dada su demanda no sólo por parte de la población migrante, sino que también de manera muy importante por parte de la población nativa que valora en ella un signo de distinción por su internacionalmente reconocida gastronomía. Son mercados que:

“(...) intentan convertir contenidos y símbolos de la etnicidad en mercancías rentables, atractivas para el conjunto de la población. Se trata de productos que sólo los inmigrantes pueden ofrecer o, como mínimo, suministrar de manera que parezcan <auténticos>”. (Solé \& Parella, 2005:62).

En cuanto al acceso a la propiedad de los negocios como factor de las estructuras de oportunidad, Waldinger distingue al menos tres elementos que inciden en el acceso a la propiedad. Por un parte, la existencia de negocios vacantes para ser usados por parte del comercio inmigrante y la competencia que exista por los mismos. En este sentido, se entiende que el abandono de la población nativa de una zona residencial hace que el comercio nativo también se desplace, liberando locales comerciales que posteriormente son usados por los empresarios inmigrantes, lo que describiría un proceso de sustitución comercial. Por otro parte, la existencia de determinadas políticas públicas orientadas a influir en los asentamientos de los inmigrantes tiene también unos consecuentes efectos en el acceso a la propiedad de negocios.

Por último, otra aproximación importante que podría enmarcarse dentro de las perspectivas interaccionistas es la que desarrolla Werbner a propósito de su estudio de la pequeña industria textil en Manchester. El acento aquí está puesto en las relaciones dentro del hogar y las más amplias redes familiares en el contexto de la empresa inmigrante, o si se quiere, la manera en que familia, capital y trabajo se interrelacionan bajo las condiciones del crecimiento y desarrollo de la comunidad inmigrante. 
Así, la idea de la comunidad inmigrante como contexto de los emprendimientos permite la producción de un encuadre para la actuación de las redes familiares y sociales, distinguiéndose dos tipos de fuerzas que constriñen las formación de los comercios en el marco de los enclaves étnicos: unas fuerzas internas, relacionadas básicamente con la familia, su crecimiento y extensión (la coincidencia entre la unidad de producción y la unidad de consumo en la empresa familiar); y unas fuerzas externas, tanto económicas como demográficas, relacionadas a su vez con el incremento de la competencia y el influjo de los inmigrantes recién llegados al nicho económico o enclave (Werbner, 1987:214-216).

A partir de la acción de estas fuerzas devienen tres procesos de implantación de los comercios étnicos en la ciudad. En primer lugar tenemos un proceso de multiplicación horizontal, a su vez compuesto de tres factores independientes: el fortalecimiento de las redes de información migrantes; el costo de establecer diferentes tipos de negocio, y; la disponibilidad de trabajo familiar. Se entiende que este proceso resulta particularmente clave y visible en las primeras fases de la migración, como cadenas empresariales que crean enclaves sub-étnicos (regionales) dentro de una industria, y donde las informaciones no son mensurables adecuadamente en términos cuantitativos, pero son claramente esenciales para la viabilidad de los pequeños negocios durante sus años formativos. En segundo lugar la extensión vertical, refiere a la participación de las firmas migrantes en distintos segmentos del proceso económico, como importación, manufactura o venta al por mayor. En tercer lugar la extensión horizontal, que remite a la diversificación de las inversiones con el objeto de reducir riesgos, abriendo negocios en otros rubros o localizaciones. En este sentido, podría aparecer como que el enclave étnico es generado particularmente en respuesta al incremento de la competencia externa entre empresas étnicas, pero también en parte es producto de la dinámica organizacional interna de las empresas y su desarrollo (Werbner, 1987:221-222).

Con todo, la flexibilidad en la comprensión del uso que se hace de los recursos étnicos queda patente en la delimitación de los contextos en que resultan pertinentes las relaciones familiares y en cuáles no. En este sentido, existen relaciones familiares que en determinados contextos resultan esenciales y que en otros resultan inviables. Es el caso del acceso a crédito por ejemplo, que según la autora es esperado como un derecho, a diferencia de otras prestaciones que quedan incalculadas. De este modo, las relaciones familiares son usadas tanto para explicar el éxito como el fracaso de estos negocios, dado que para el éxito del comercio étnico las relaciones contractuales en la esfera comercial deben estar claramente especificadas y estrictamente limitadas (Werbner, 1987:228-229).

\section{El modelo del encajado mixto}

Si bien este modelo explicativo de las economías étnicas podría ser entendido ampliamente como una perspectiva interaccionista, en términos 
de que no se restringe al análisis de los llamados recursos étnicos, presenta algunas particularidades que hacen relevante presentarlo separadamente. El origen de esta teoría se encuentra básicamente en el intento de Kloosterman y otros por dar cuenta de las especificidades del caso europeo. Esta perspectiva enfatiza los contextos de recepción, en particular lo que refiere a los aspectos jurídicos-normativos como elementos claves en la formación de las economías étnicas (Kloosterman, Van der Leun et al., 1999; Arjona, Checa et al., 2007:5). Según Light, la teoría del encajado mixto introduce en la perspectiva del interaccionismo una estructura de oportunidad con un aspecto normativo y otro de demanda. De este modo, se entenderá que las economías étnicas dependen de la adecuación entre lo que los grupos pueden ofrecer y lo que está permitido que ofrezcan, no entre la demanda del consumidor y lo que los grupos pueden ofrecer. Lo que se establece entonces es una crítica al supuesto economicismo que caracterizaría el modelo establecido por Waldinger, centrado exclusivamente en la demanda de los consumidores (Light, 2007:54)

En este sentido, se entiende que los mercados de inmigrantes emprendedores suelen ser altamente competitivos entre co-nacionales o co-étnicos, competencia que se centra básicamente en el precio más que en la calidad. Consecuentemente, estos comercios acceden a pequeños márgenes de ganancia, lo que influye en que permanezcan por poco tiempo en actividad. Esto a su vez, empujaría a los empresarios y trabajadores involucrados hacia actividades de carácter informal (Rath \& Kloosterman, 2000:660-661). A partir de allí, se han identificado tres modelos de incrustación de los emprendimientos inmigrantes, marcados por el marco institucional en que se insertan (Kloosterman, 2001): a) "Neo-American Model", presente en EEUU Australia, Irlanda e Inglaterra, que coincide con los postulados de la corriente interactiva, y donde optar por la auto-ocupación no se produce a efectos de una carencia al nivel de las oportunidades, sino una estrategia que permite obtener mayores ingresos y mejores posibilidades de movilidad laboral ascendente (Parella, 2005:264); b) "Rhineland Model" (presente en Austria, Francia, Luxemburgo y Alemania). Da cuenta de un marco institucional precisamente contrario al anterior, esto es, un contexto de altos salarios a cambio de alto desempleo, cuestión que deriva en un importante control del flujo migratorio por parte del Estado y grandes trabas para la inserción laboral de los inmigrantes. Se trata de un modelo con estructura dicotómica (insider/outdsider) (Arjona, Checa et al., 2007:6); c) “Nordic Model” (presente en Dinamarca y Suecia). En este modelo se observa un control importante de la economía por parte del Estado, parecido al modelo anterior pero la diferencia está en la aplicación de políticas activas en el mercado de trabajo, expansión del estado de bienestar e igualdad de género. La consecuencia es una menor presencia de empresariado étnico, una mayor regulación de las relaciones laborales y la aplicación de políticas de igualdad que fomentan el que los inmigrantes opten por insertarse en la economía abierta (Arjona, Checa et al., 2007:6). 


\section{Consideraciones finales}

La producción teórica que hemos revisado acerca de la noción de economía étnica y comercio inmigrante tiene un marcado acento en un contexto migratorio que podríamos caracterizar como migración "Sur-Norte". La consecuencia de ello puede estribar en la dificultad para aplicar algunos de sus marcos de análisis a las realidades de una migración intrarregional como la que se observa en los países de América del Sur. Nos referimos particularmente a las dimensiones relativas a las características de la estructura de oportunidades (marcos jurídico-institucionales) en que tienen lugar dichos emprendimientos. Sin embargo, creemos que estos enfoques iluminan importantes aspectos escasamente desarrollados en el marco de la aún incipiente investigación sobre los contemporáneos flujos migratorios en este continente.

Por una parte, el análisis de los marcos regulatorios que configuran el contexto de recepción de la población migrante constituye un campo poco investigado y insuficientemente puesto en relación con las vidas cotidianas de las migrantes. Un ejemplo de esta problemática es el caso chileno, donde la normativa que regula la vida de los migrantes se mantiene aún fundada en un marco legal creado en la década de los 70, y que por lo mismo no asume la trasformación de la dinámica migratoria y el hecho de que el país haya venido a convertirse en un polo atractor de inmigración. Por otro lado, la flexibilización de los mercados de trabajo en el marco de reformas económicas liberales en la región, coloca a los inmigrantes es posición muchas veces desventajosa (cuando no discriminados por cuestiones relativas al origen étnico o nacional) que les fuerza a ocupar los sectores de empleo menos calificados y peor remunerados, colocando la alternativa de convertirse en micro-empresarios como casi la única posibilidad de acceder a una cierta movilidad social.

Donde también pueden ser muy productivas las perspectivas teóricas expuestas es lo que refiere a la investigación del proceso económico interno a la formación de estos nuevos emprendimientos que empezamos a observar en nuestras grandes ciudades. ¿Cuáles son las biografías o historias migratorias que explican la formación de estos enclaves o espacios de concentración?, ¿qué recursos o capitales culturales, sociales o financieros se ponen en juego?, ¿cuándo y de qué tipo son las relaciones o vínculos que establecen con origen? ¿qué transformaciones en el paisaje urbano van produciendo?, ¿se da lugar a la revitalización de espacios urbanos deteriorados como sugiere la experiencia europea?, ¿de qué manera introducen diversidad cultural en un paisaje urbano pretendidamente homogéneo?, ¿de qué modos la relación con la población local se transforma en una relación de clientela? Son todas preguntas que deben ser planteadas frente a un fenómeno como éste en notoria expansión. Con todo, debemos intentar acercarnos sin rigideces, quizás liberando a la noción de economía étnica de esa clausura con que suele presentársela dentro de único colectivo o minoría, para así dar cuenta de la forma cómo estos emprendimientos dialogan con la sociedad en que se insertan, convirtiéndola en un espacio económico 
muchas veces pujante y deseado por empresarios no sólo migrantes sino también nativos, que observan la inauguración de lo étnico como objeto de un comercio, o si se quiere, que transforman lo étnico en objeto de consumo. 
Polis, Revista de la Universidad Bolivariana, Volumen 10, $N^{\circ} 29,2011$

\section{Notas}

${ }^{1}$ La idea de esta etnificación de los espacios urbanos ha sido abordados por investigadores en algunos barrios de la ciudad de Paris, Francia (Toubon \& Messamah, 1990; Simon, 1997).

${ }^{2}$ Esta relación entre el carácter del mercado de trabajo y la economía étnica o de enclave se encontraba ya presente en el clásico trabajo de Wilson Portes acerca del enclave cubano en Miami. En dicha investigación se establecía como hipótesis el que a partir de las variables diferenciales de los inmigrantes en tres mercados de trabajo (primario, secundario y enclave), se esperaba encontrar diferencias en el prestigio ocupacional, en la estabilidad económica, satisfacción ocupacional y de ingreso, percepción y experiencias de discriminación, interacción con angloamericanos, y otras variables relacionadas entre inmigrantes empleados en empresas centrales y periféricas (Wilson \& Portes, 1980:306).

${ }^{3}$ Solidaridad étnica entendida como una suerte de etnicidad reactiva a las situaciones de discriminación o desventaja en el mercado laboral (Light \& Rosenstein, 1995; Riesco, 2003:108) 


\section{Bibliografía}

Aldrich, Howard (1975), "Ecological succession in racially changing neighbourhoods: a review of the literature.” Urban Affairs Quarterly 10: 327-348.

Aldrich, Howard \& Albert Reiss (1976), "Continuities in the study of ecological succession; changes in the race composition of neighbourhoods and their businesses.” American Journal of Sociology 81: 846-866.

Aramburu, Mikel (2002), "Los comercios de inmigrantes extranjeros en Barcelona y la recomposición del 'inmigrante' como categoría social.” Scripta Nova. Revista Electrónica de Geografía y Ciencias Sociales. Vol VI, no 108. http://www.ub.es/geocrit/sn/sn-108.htm

Arjona, Ángeles (2006), Los colores del escaparate. Emprendedores inmigrados en Almería. Icaria, Barcelona.

Arjona, Ángeles \& Juan Carlos Checa (2007), “Ubicación espacial de los negocios étnicos en Almería. ¿Formación de enclaves económicos étnicos?” Estudios Geográficos LXVIII (263): 391-415.

Arjona, Ángeles, Juan Carlos Checa, et al. (2007), “Discusión teórica e implicaciones transnacionales de las economías étnicas”. V Congreso sobre la Inmigración en España. Migraciones y desarrollo humano, Valencia. http://www.adeit.uv.es/inmigracion2007/index.php

Beltrán, Joaquín (2000), “Empresa familiar. Trabajo, redes y familia en el colectivo chino.” OFRIM/Suplementos Nº6: 129-153.

Beltrán, Joaquín, Laura Oso, et al. (2007), “Un campo de estudio para el empresariado étnico en España”. En: J. Beltrán, L. Oso \& N. Ribas Empresariado étnico en España. Fundación CIDOB, Barcelona.

Bonacich, Edna (1973), “A theory of middleman minorities.” American Sociological Review 38: 583-94.

Ídem (1987), “'Making it' in America: a social evaluation of the ethics of immigrant entrepreneurship.” Sociological Perspectives 30: 446-66.

Bonacich, Edna \& J Modell (1980), The economic basis of ethnic solidarity: small business in the japanese american community. University of California Press, Berkeley.

Bourgeois, Philippe (1995), In Search of Respect: Selling Crack in El Barrio. Cambridge University Press, Cambridge.

Buckley, Mónica (1998), “Inmigración y comercio en Madrid. Nuevos negocios para nuevas gentes.” Anales de Geografía de la Universidad Complutense $\mathrm{n}^{0} 18:$ 283-297. 
Cebrián de Miguel, Juan \& M M Isabel Bodega (2002), “El negocio étnico, nueva fórmula de comercio en el casco antiguo de Madrid. El Caso de Lavapiés.” Estudios Geográficos, LXIII, 248/249: 559-580.

Garcés, Alejandro (2005), “Espacios comerciales”. En: VV.AA. Espacios urbanos e inmigración en el Madrid del siglo XXI. Casa Encendida, Madrid.

Ídem (2006), “Configuraciones espaciales de lo inmigrante: usos y apropiaciones de la ciudad.” Papeles del CEIC n ${ }^{\circ}$ 20, CEIC (Centro de Estudios sobre la Identidad Colectiva), Universidad del País Vasco: 1-34. http:/ /www.identidadcolectiva.es/pdf/20.pdf

Ídem (2007), "Entre lugares y espacios desbordados: formaciones urbanas de la migración peruana en Santiago de Chile.” Serie Documentos. Facultad de Ciencias Sociales - Universidad Central N²2: Abril de 2007. 522.http://www.fcsucentral.cl/varios/files/file/publicaciones/ antropologiaok.pdf

Ídem (2010), Movimientos y localizaciones: espacios públicos y economías de la migración peruana en Santiago de Chile. Tesis Doctoral. Departamento de Antropología Social, Universidad Autónoma de Madrid, Madrid.

García Ballesteros, Ana, Emilia García, et al. (2006), Inmigrantes emprendedores en la Comunidad de Madrid. Madrid: Consejería de Economía e Innovación Tecnológica. Comunidad de Madrid.

Gómez, Paloma (2006), Inmigración y Comercio en Madrid: Empresarios, Consumidores, Trabajadores y Vecinos. Observatorio de las Migraciones y de la Convivencia Intercultural de la Ciudad de Madrid, Madrid.

Ídem (2007), “La incorporación de pequeños empresarios inmigrantes al ámbito comercial madrileño: oportunidades y estrategias”. V Congreso sobre la Inmigración en España. Migraciones y desarrollo humano, Valencia.

Kloosterman, Robert (2001), “Immigrant Entrepreneurship and the institutional Context: A Theoretical Exploration”. En: J. Rath Immigrant Business. The economic, politic and social environtment. Centre for Research in Ethnic Relations, Warwick.

Kloosterman, Robert, J Van der Leun, et al. (1999), “Mixed- embeddedness: (In)formal economic activities and immigrant businesses in the Nethernlands.” International Journal of Urban Research 23 (2): 252-266.

Light, Ivan (1972), Ethnic Enterprise in America: Business and Welfare among Chinese, Japanese, and Blacks. University of California Press, Berkeley. 
Ídem (1984), “Immigrant and ethnic enterprise in North America.” Ethnic and Racial Studies 7: 195-216.

Ídem (2007), “Economías étnicas”. En: J. Beltrán, L. Oso \& N. Ribas Empresariado étnico en España. CIDOB, Barcelona.

Light, Ivan \& Steven Gold (2000), Ethnic Economies. Academic Press, San Diego.

Light, Ivan \& Carolyn Rosenstein (1995), Race, Ethnicity and Entrepreneurship in Urban America. Aldine de Gruyter, New York.

Min, P.G (1984), “From white-collar occupations to small business: korean’s occupational adjustment.” Sociological Quarterly 25: 333-352.

Ídem (1996), “The entrepreneurial adaptation of korean immigrants”. En: S. Pedraza \& R. Rumbaut Origins and destinies. Immigration, race and ethnicity in America. Wadsworth, Belmont.

Monnet, Nadja (2002), La formación del espacio público. Una mirada etnológica sobre el Casc Antic de Barcelona. Catarata, Barcelona.

Moreras, Jordi (1999), Musulmanes en Barcelona. Espacios y dinámicas comunitarias. CIDOB, Barcelona.

Nelson, Candace \& Marta Tienda (1985), “The structuring of hispanic ethnicity: historical and contemporary perspectives.” Ethnic and Racial Studies 8: 49-74.

Parella, Sònia (2005), “Estrategias de los comercios étnicos en Barcelona, España.” Política y Cultura 23: 257-275.

Portes, Alejandro \& Robert Bach (1985), Latin journey: cuban and mexican immigrants in the United States. University of California Press, Berkeley.

Portes, Alejandro \& Jözsef Böröcz (1998), “Migración Contemporánea. Perspectivas teóricas sobre sus determinantes y modalidades de incorporación”. En: G. Malgesini Cruzando fronteras. Migraciones en el sistema mundial. Icaria, Barcelona.

Portes, Alejandro \& Leif Jensen (1989), “The enclave and the entrants: patterns of ethnic enterprise in Miami before and after Mariel.” American Sociological Review 54 (6): 929-949.

Rath, Jan \& Robert Kloosterman (2000), “Outsiders’ Business: A Critical Review of Research on Immigrant Entrepreneurship.” International Migration Review 34 (3): 657-681.

Riesco, Alberto (2003), “Enclaves y economías étnicos desde las relacio- 
nes salariales.” Cuadernos de Relaciones Laborales 21 (2): 103-125.

Sanders, Jimy \& Victor Nee (1987), "Limits of ethnic solidarity in the enclave economy.” American Sociological Review 52 (745-73).

Sassen, Saskia (1991), The global city. New York, London, Tokyo. New Jersey: Princeton University Press.

Serra, Pau (2006), El comercio étnico en el distrito de Ciutat Vella Barcelona. Fundación 'La Caixa', Barcelona.

Simon, Patrick (1997), "Les usages socieaux de la rue dans un quartier cosmopolite.” Espaces et sociétés 90-91: 43-68.

Solé, Carlota \& Sònia Parella (2005), Negocios étnicos. Los comercios de los inmigrantes no comunitarios en Cataluña. CIDOB, Barcelona.

Sow, P (2004), "Prácticas transnacionales y espacios de acción de los senegaleses en España”. En: A. Escrivá \& N. Ribas Migración y desarrollo. Estudio sobre remesas y otras prácticas transnacionales en España. Consejo Superior de Investigaciones Científicas, Córdoba.

Stefoni, Carolina (2005), “Comunidades transnacionales y la emergencia de nuevas oportunidades económicas. De empleados a microempresarios.” Persona y Sociedad XIX (N³): 183-197.

Ídem (2008), “Gastronomía peruana en las calles de Santiago y la construcción de espacios transnacionales y territorios”. En: S. Novick Migraciones en América Latina. Catálogos, Buenos Aires.

Tienda, Marta \& Rebeca Raijman (2000), "Immigrants income packaging and invisible labor force activity.” Social Science Quarterly 81: 291-310.

Torres Pérez, Francisco (2008), “Los nuevos vecinos en la plaza. Inmigrantes, espacios y sociabilidad pública.” AIBR, Revista de Antropología Iberoamericana 3 (3): 336-397.

Toubon, Jean Claude \& Klelifa Messamah (1990), Centralité immigrée. Le quartier de la Goutte d'Or. L'Harmattan-CIEMI, Paris.

Waldinger, Roger (1986), “Changing leaders and musical chairs: ethnicity and opportunity in post-industrial New York.” Politics and Society 15 (369401).

Waldinger, Roger, Howard Aldrich, et al. (1990), “Opportunities, group characteristics, and strategies”. En: R. Waldinger, H. Aldrich \& R. Ward Ethnic Entrepreneurs. Immigrant Business in Industrial Societies. SAGE Publications, Newbury Park. 
Werbner, Pnina (1987), "Enclave economies and family firms: Pakistani traders in a British city”. En: J. Eades Migrants, Workers, and the Social Order. Tavistock, London \& New York.

Ídem (1999), “What colour 'success'? Distorting value in studies of ethnic entrepreneurship.” Sociological Review 47 (3): 548-579.

Wilson, Kenneth \& W. Allen Martin (1982), "Ethnic enclaves: a comparison of the cuban and black economies in Miami." American Journal of Sociology 88: 135-60.

Wilson, Kenneth \& Alejandro Portes (1980), "Immigrant enclaves: an analysis of the labor market experiencies of cubans in Miami." American Journal of Sociology 86 (2): Sep., 1980. 295-319.

Recibido: 20.05.2011

Aceptado: 06.07.2011 